\title{
Ballistic excited state dynamics revealed by polarized fs-XANES
}

\author{
Roseanne J. Sension ${ }^{1,2,3,{ }^{*}, \text { Nicholas A. Miller }}{ }^{1}$, Aniruddha Deb $^{1}$, Roberto Alonso-Mori ${ }^{4}$, \\ James M. Glownia ${ }^{4}$, and James E. Penner-Hahn ${ }^{1,2}$ \\ ${ }^{1}$ Department of Chemistry, University of Michigan, Ann Arbor Michigan, 48109, USA \\ ${ }^{2}$ Biophysics, University of Michigan, Ann Arbor, Michigan, 48109, USA \\ ${ }^{3}$ Department of Physics, University of Michigan, Ann Arbor, Michigan, 48109, USA \\ ${ }^{4}$ Linac Coherent Light Source, SLAC National Accelerator Laboratory, Menlo Park, CA 94025, USA
}

\begin{abstract}
Polarized time-resolved X-ray absorption near edge structure (XANES) is used to characterize the sequential ballistic excited state dynamics of two $\mathrm{B}_{12}$ vitamers: cyanocobalamin and adenosylcobalamin. Excitation at $550 \mathrm{~nm}$ and $365 \mathrm{~nm}$ is used to resolve axial and equatorial contributions to the excited state dynamics.
\end{abstract}

\section{Introduction}

Excitation of a photoactive molecule initiates structural rearrangements that determine the photochemical fate of the molecule. With the development of femtosecond X-ray free electron lasers it has become possible to interrogate directly the local structural changes that take place at the earliest stages of photochemical excitation. Although it is often possible to fit the observations using a kinetic model, there is little reason to expect such a model to accurately account for the dynamics on the fastest time scales. Alternatively, the earliest times following photoexcitation can be treated as the molecular trajectory of a coherent wave packet. Oscillatory behavior in well-defined vibrational modes can make the identification of coherent dynamics straightforward. However, coherent ballistic motion on a potential energy surface, particularly a reactive surface or one defined by large structural changes, will not always be characterized by oscillations in well-defined vibrational modes.

We have used femtosecond time-resolved X-ray absorption near edge structure (fsXANES) at the Co K-edge to characterize the excited state dynamics of vitamin $\mathrm{B}_{12}$ (cyanocobalamin, $\mathrm{CNCbl}$ ) and coenzyme $\mathrm{B}_{12}$ (adenosylcobalamin, AdoCbl) at the earliest times following photoexcitation. The femtosecond evolution of the XANES spectrum was monitored following excitation at $540-550 \mathrm{~nm}$ and excitation at $365 \mathrm{~nm}$. The two orthogonal transitions permit full deconvolution of the $\mathrm{x}, \mathrm{y}$, and z-polarized excited-state XANES difference spectra as the molecule undergoes evolution from the initial structure to the relaxed excited state minimum energy structure. Our data support a dynamic model in which the molecule can be viewed as following a ballistic trajectory on a multidimensional potential energy surface for the first few hundred fs after excitation.

\footnotetext{
*Corresponding author: rsension@umich.edu
} 

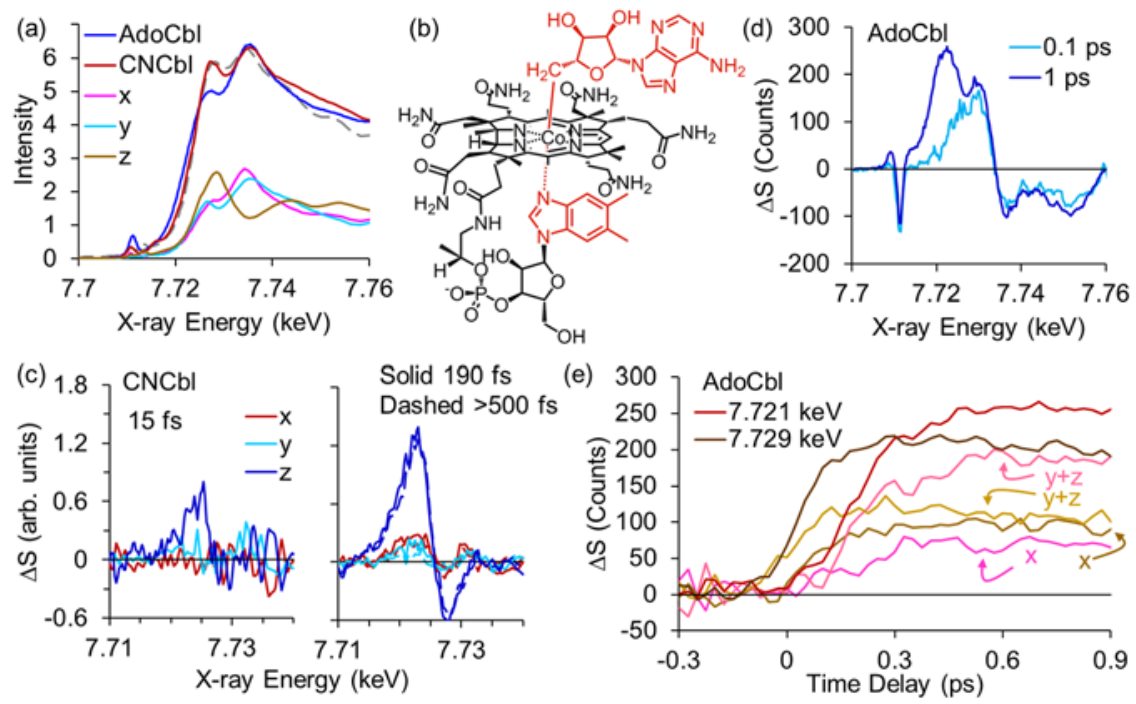

Fig. 1. (a) Experimental XANES spectrum of AdoCbl and $\mathrm{CNCbl}$ along with the simulated spectrum of $\mathrm{CNCbl}$ (gray dashed line) [5]. The individual $x, y$, and $z$ contributions to the simulated spectrum are also plotted. (b) The structure for AdoCbl. $\mathrm{CNCbl}$ has a $-\mathrm{C} \equiv \mathrm{N}$ group as the upper axial ligand.

(c) The $x, y$, and $z$ contributions to the XANES difference spectrum of $\mathrm{CNCbl}$ at three key time delays

[3]. (d) The isotropic XANES difference spectrum following excitation of AdoCbl in water at 540 $\mathrm{nm}$. (e) Time evolution of the XANES difference at the two peaks observed in (d).

\section{Experimental methods}

XANES difference spectra, $\Delta S(t)=S_{\text {on }}(t)-S_{\text {off }}$, were obtained for time delays between $300 \mathrm{fs}$ and 1 ps using the XPP instrument of the x-ray free electron laser LCLS at SLAC [13]. The $\mathrm{CNCbl}$ or AdoCbl sample was dissolved in ultrapure deionized water and pumped through a glass nozzle to achieve a stable $50 \mu \mathrm{m}$ diameter jet of solution. The X-ray beam and laser beam travel in a nearly collinear geometry $\left(\sim 1^{\circ}\right.$ crossing angle $)$ and were overlapped with the sample about $500 \mu \mathrm{m}$ below the nozzle. The optical pump pulse $(\sim 50$ fs fwhm) was centred at $550 \mathrm{~nm}, 540 \mathrm{~nm}$, or $365 \mathrm{~nm}$. The X-ray probe pulse ( 40 fs fwhm) was tuned from $7.7 \mathrm{keV}$ to $7.8 \mathrm{keV}$ and cobalt $\mathrm{x}$-ray fluorescence was used as a measure of absorption. The pump pulsewas polarized parallel or perpendicular to the probe pulses.

\section{Results}

The ground state XANES of CNCbl and AdoCbl obtained from the laser-off measurements are plotted in Fig. 1(a). The difference spectrum, $\Delta S(t)$, is sensitive to shifts in the XANES edge resulting from changes in the ligation around the central cobalt atom. Qualitatively, expansion of the cavity around the cobalt results in a red-shift of the edge and contraction results in a blue-shift of the edge. Polarization anisotropy has long been exploited in ultrafast optical measurements [4]. Decomposition of the X-ray transient difference signal is achieved using the same theory. In this case the X-ray transition can be decomposed into any set of orthogonal molecule-fixed coordinates. The obvious choice is $a$ parallel to the transition dipole initially pumped and $b+c$ perpendicular to this transition dipole direction. The signals obtained with the polarization of the optical pulse parallel and perpendicular to the polarization of the $\mathrm{x}$-ray pulse are: 


$$
\left.\begin{array}{c}
\Delta S_{\|}=0.6 \Delta S_{a}+0.2 \Delta S_{b}+0.2 \Delta S_{c} \\
\Delta S_{\perp}=0.2 \Delta S_{a}+0.4 \Delta S_{b}+0.4 \Delta S_{c}
\end{array}\right\} \Rightarrow \begin{aligned}
& \Delta S_{a}=2 \Delta S_{\|}-\Delta S_{\perp} \\
& \Delta S_{b+c}=3 \Delta S_{\perp}-\Delta S_{\|}
\end{aligned}
$$

Ultrafast polarized XANES measurements of CNCbl allow separation of the in-plane $(x$ and $y$ ) contributions to $\Delta S(t)$ from the out-of-plane contribution $(z)$. The transitions excited at $550 \mathrm{~nm}$ and $365 \mathrm{~nm}$ are polarized approximately orthogonally in the plane of the corrin ring and the isotropic XANES difference spectra are essentially independent of excitation wavelength. Thus, $\Delta S_{x}$ and $\Delta S_{y+z}$ determined following $550 \mathrm{~nm}$ excitation and $\Delta S_{y}$ and $\Delta S_{x+z}$ determined following $365 \mathrm{~nm}$ excitation can be used to separate the axial and equatorial changes in structure. The results of this decomposition are shown in (Fig. 1(c)). The magnitude of the shift demonstrates that evolution on the excited state surface is ballistic rather than kinetic [3]. A kinetic model results in a structure for the intermediate state at $190 \mathrm{fs}$ that is physically unrealistic, while a ballistic model is consistent with theoretical simulations. The measured changes in the $x, y$, and $z$ directions provide a qualitative picture of the excited state dynamics. The initial $\pi \rightarrow \pi^{*}$ excitation increases the diameter of the equatorial cavity. This is followed by significant elongation of the axial bonds, reaching maximum extension $190 \mathrm{fs}$ after excitation. The axial bonds overshoot equilibrium bond lengths for the relaxed excited state by $\sim 0.025 \AA$. As the molecule relaxes to the equilibrium excited state, the axial bonds and diameter of the corrin cavity contract slightly [3].

The initial dynamics following excitation of AdoCbl are also ballistic rather than kinetic. Sequential changes in the $y$, then $x$ and finally $z$ directions are revealed by the femtosecond XANES difference spectra (Fig. 1(d,e)). The initial peak in $\Delta S(t)$ at 7.729 $\mathrm{keV}$ is $y+z$ polarized, but dominated by the $y$ contribution. The shift of the $x$-polarized contribution follows in ca. $75 \mathrm{fs}$. The shift of the XANES edge characterized by the peak in $\Delta S(t)$ at $7.721 \mathrm{keV}$ is dominated by increases in the axial bond lengths ca. $125 \mathrm{fs}$ after the initial excitation. As for $\mathrm{CNCbl}$, the changes in the AdoCbl XANES difference spectrum cannot be accounted for using a simple kinetic model.

This work was supported by a grant from the National Science Foundation NSF-CHE 1464584 to RJS. Use of the Linac Coherent Light Source (LCLS), SLAC National Accelerator Laboratory, is supported by the U.S. Department of Energy, Office of Science, Office of Basic Energy Sciences under Contract No. DE-AC02-76SF00515.

\section{References}

1. M. Chollet, R. Alonso-Mori, M. Cammarata, D. Damiani, J. Defever, J. T. Delor, Y. P. Feng, J. M. Glownia, J. B. Langton, S. Nelson, K. Ramsey, A. Robert, M. Sikorski, S. Song, D. Stefanescu, V. Srinivasan, D. L. Zhu, H. T. Lemke, and D. M. Fritz, J. Synchrotron Rad. 22, 503 (2015).

2. N. A. Miller, A. Deb, R. Alonso-Mori, B. D. Garabato, J. M. Glownia, L. M. Kiefer, J. Koralek, M. Sikorski, K. G. Spears, T. E. Wiley, D. Zhu, P. M. Kozlowski, K. J. Kubarych, J. E. Penner-Hahn, and R. J. Sension, J. Am. Chem. Soc. 139, 1894 (2017).

3. N. A. Miller, A. Deb, R. Alonso-Mori, J. M. Glownia, L. M. Kiefer, A. Konar, L. B. Michocki, M. Sikorski, D. L. Sofferman, S. Song, M. J. Toda, T. E. Wiley, D. L. Zhu, P. M. Kozlowski, K. J. Kubarych, J. E. Penner-Hahn, and R. J. Sension, J. Phys. Chem. A 122, 4963 (2018).

4. G. R. Fleming, Chemical Applications of Ultrafast Spectroscopy, (Oxford University Press, Oxford, 1986).

5. P. Lodowski, M. Jaworska, K. Kornobis, T. Andruniow, and P. M. Kozlowski, J. Phys. Chem. B 115, 13304 (2011). 